\title{
Editorial
}

\section{Announcement of a special virtual issue on the EuroGRAPHENE program}

The Carbon journal is pleased to announce the publication of a virtual special issue on research funded by the Euro- GRAPHENE program of the European Science Foundation. In order to ensure rapid publication, the contributed primary research articles have already appeared in regular Carbon volumes over the course of 2014, and are now compiled together as a virtual special issue on the journal website. 1 The present article by our team of guest editors serves as an introduction to this special issue, and also a commentary on the graphene field and the important role played by the EuroGRAPHENE program.

Ten years ago a revolution began that has taken the solid state physics and materials science communities by storm: the discovery of how to prepare and process graphene, the first of a large family of monoatomic, 2-dimensional materials. This discovery and subsequent analysis of this material led to amazing discoveries at a fundamental level, such as the presence of massless charge carriers in graphene, whose behavior is governed by quantum electrodynamics. Geim and Novoselov were awarded the Nobel Prize in Physics 2010 for these discoveries in a material that is "all surface and no bulk". The unusual physical properties of this material - strength, high carrier mobility, etc. have also raised hopes that a new era in materials science applications has dawned, in electronic devices in particular, but also in other fields. Ten years and tens of thousands of publications later, the struggle to fully understand graphene's physical properties either as a free-standing entity or in contact with other materials, and the race to potential applications shows no sign of slowing down. Moreover, the prospect of combining graphene with other truly 2D materials such as hexagonal boron nitride or single/multilayers of transition metal chalcogenides offers the prospect of creating a new class of 2-dimensional composite structures that may bring new functionalities in electronic data processing and storage devices, to name only one possible application. Graphene's properties were first discovered in Europe, by Geim and Novoselov at the University of Manchester. Immediately afterwards, research on graphene flourished worldwide and many countries, such as South Korea or Singapore, rapidly established strong research activities in this field, both in academic and industrial labs, launching 
ambitious research programs on graphene. In Europe, one of the first coordinated efforts on graphene was initiated by the European Science Foundation, which in 2009 launched the EuroGRAPHENE program.

EuroGRAPHENE was conceived in mid-2008 as the first multinational European initiative to study various aspects of graphene from a fundamental as well as applications-oriented point of view, and started operation at the end of 2010. Within seven collaborative research projects, 43 principal investigators and associated partners, EuroGRAPHENE addressed graphene-related issues, from graphene-organic transistors and optoelectronic devices through graphene-ferromagnetic junctions to spin-entangled states in hybrid graphene systems. Work within these projects was funded by the national research funding agencies from 2010 through 2014, and the contents of the EuroGRAPHENE program across national and institutional boundaries was discussed at several large meetings and numerous small workshops. Intense collaborations have emerged from the EuroGRAPHENE program and continue to be active beyond its conclusion.

This special issue of CARBON contains scientific papers resulting from work within EuroGRAPHENE's projects. This is only a small selection among the many publications that have emerged and are still in the process of being submitted from those projects; moreover, in line with the main audience of CARBON, many papers in this volume deal with specific aspects of graphene research, e.g. the preparation and processing of different kinds of graphene. The ensemble of these articles reflects the enormous variety of scientific fields where graphene can play a role, from physics to chemistry, surface science, nanotechnology and more.

Among the topics covered in this volume, silicon carbide as the singly most important substrate for high quality epitaxial growth plays a major role. Apart from the ubiquitous growth on the hexagonal silicon-terminated $\mathrm{SiC}(0001)$ surface, the growth of single and bilayer graphene has been performed on nonpolar surfaces of hexagonal $\mathrm{SiC}$ as well as on the polar cubic (100) surface. Intercalation of gases (e.g. hydrogen) and metals has been a very active subfield of graphene research, and a few representative studies appear in this volume. Interest has also concentrated on precursors to graphene preparation, such as graphene oxide and its photo-induced or electrochemical reduction. The improvement of organic and inorganic composites using graphene oxide, as 
example to improve battery storage capacity or photochemical water-splitting are other examples in this volume, investigated from an applications-oriented viewpoint. The property of graphene nanoribbons is another exciting topic in graphene research, since these are thought to be the solution to one of graphene's basic problems in electronic switching applications - the absence of a sizeable fundamental band gap.

Overall, these papers highlight one of the most exiting features of graphene research, i.e. the strong intermixing of fundamental science and enormous technological interests. Graphene nanoribbons and nanodots, for example, are an ideal playground to study the optical and electronic properties of graphene using theory and modelling; on the other side, they can give a key to the highly desired production of graphene-based transistors. Graphene growth on $\mathrm{SiC}$ is a fascinating problem of surface science on one side; on the other, achieving a complete control of this physical process would allow to obtain graphene of the highest quality on non-conductive substrates. This dual interest is present also if we focus on graphene chemistry. Graphene oxide (GO) is a "strange" chemical object, whose exact structure is still far from being completely understood, and controlled. Once an underdog in the graphene community, it is now the most processable and tunable among the 2D materials, and is used to produce highly performing sensors, batteries or composites, as some papers of this issue demonstrate. The EuroGRAPHENE program ended in 2013, and was rated as "highly successful" by an external review panel of experts. Citing their words:

"EuroGRAPHENE has created and helped in shaping a closely collaborating community of researchers in graphene science and technology in Europe. This community has grown about ten times since the start of the program. Projects benefitted from the complimentary expertise of partners in different countries and from interactions between theory and experiments. Overall, the total number of publications, around 300, is impressive."

However, in our opinion, the most important result of the EuroGRAPHENE initiative was not only the large number of publications (including nine patents) obtained. The program has served as a model for a larger collaboration encompassing many European nations. A main criticism raised was the relative short time-span of the program funding - only 3 years. In 2013, after a tough selection process, the European commission decided to launch another project on graphene, the "GRAPHENE Flagship" 
(http://graphene-flagship.eu/) - much more ambitious, larger and longer than anything ever tried before. This cooperation involves about 140 research groups throughout Europe, is planned to last for ten years, and dedicated to transforming the scientific revolution of graphene into real, useful technology. The EuroGRAPHENE program initiated by the European Science Foundation can be rightfully seen as the forerunner to the GRAPHENE Flagship, having demonstrated by the feasibility of such a cooperation that, even under stiff international competition, European research can be at the forefront in the field, developing new ways to grow and process this fascinating material, and use it in different applications fields. With this special issue, we want to give a glimpse of what EuroGRAPHENE participants have worked and continue to work on, and a panoramic view of the research lines generated from that first, seminal European research project on graphene.

Vincenzo Palermo

ISOF-CNR, via Gobetti 101 , I-40129 Bologna, Italy

Antonino La Magna

IMM-CNR, VIII Strada, 5, 95121 Catania, Italy

Ana Helman

European Science Foundation, 1, quai Lezay Marnésia,

BP 90015, F-67080 Strasbourg Cedex, France

Robert Hurt Editor-in-Chief, Carbon

Karsten Horn

Department of Physical Chemistry, Fritz-Haber-Institut der Max-Planck-Gesellschaft, Faradayweg 4-6, 14195 Berlin, Germany 